\title{
Reaction Times for Esport Competitors and Traditional Physical Athletes are Faster than Noncompetitive Peers
}

\author{
ANH LUU, AVORY WINANS, REMA SUNIGA, and VICKI A. MOTZ ${ }^{1}$, Ohio Northern University, Ada, OH, USA.
}

ABSTRACT. Reaction time (RT), time to respond to a stimulus, has been shown to be faster among traditional physical athletes and esport competitors than nonathletes/noncompetitors; however, no comparison has been made between traditional physical athletes and esport competitors. This research examined RTs of healthy 18 to 22 year-old college football athletes, esport competitors, and a control group ( $n=12$ for each group). RT (ms) to visual (color cue test, ruler drop test), auditory (sound cue test), and tactile stimuli (probe grabbing test) was collected in duplicate. RTs for individual tests and calculated composite RTs were compared between groups by ANOVA and post hoc $t$-tests. RTs to auditory stimuli were significantly slower than to visual or tactile stimuli $\left(F_{(140,3)}=286.5, p=0.0000\right)$. Esport competitors significantly outperformed noncompetitive controls in probe grabbing $(p=0.0175)$ and ruler drop tests $(p=0.0016)$. Football players had similar faster RTs in probe grabbing $(p=0.0002)$ and ruler drop tests $(p=0.0013)$ compared to controls. Esport competitors also had significantly faster RTs in the color cue test than controls $(p=0.05)$. Although esport competitors and football athletes had faster composite RTs than controls ( $p=0.0042$ and $p=0.0104$, respectively), RTs between esport competitors and football athletes were not significantly different. A trend was seen in that esport competitors had faster RTs than football athletes in all tests except probe grabbing. Involvement in esports or football is positively correlated with faster RT, although it is not demonstrated whether play improves RT or those with inherently faster RTs tend to excel in activities requiring rapid response.

\section{INTRODUCTION}

Reaction time (RT) is the time interval between a signal and the reaction to it (Radák 2018). Response times to specific stimuli have been shown to be age-dependent (improving from ages 3 to 15, not changing significantly to age 30 , and deteriorating thereafter) (Bucsuházy and Semela 2017), but not gender-dependent (Woods et al. 2015) or IQdependent (Aktas 2019). Rate of eye movement in response to visual stimuli is directly proportional to hand-eye coordination (Dean et al. 2011).

RT can be measured by several methods, including the ruler drop test (Latorre-Roman et al. 2018), measuring reaction to a visual stimulus (Bucsuházy and Semela 2017), a sound stimulus (Kemp 1984), or a tactile stimulus (Hernández et al. 2005); test protocols will be elucidated in the methods section.

Esport competitors, gamers who play genres that are considered action esport games (such as first-person shooter (FPS) and massive online battle arena (MOBA) games) had faster RTs on the trail-making (visual/cognitive) test than those

${ }^{1}$ Address correspondence to Vicki A. Motz, Ohio Northern University, 525 South Main St., Ada, OH 45801, USA. Email:v-motz@onu.edu without a gaming background (Kowal et al. 2018). Furthermore, players of these types of competitive action video games had quicker RTs in auditory and visual perceptual decision making tasks than players without that exposure (Green et al. 2010). Gozli et al. (2014) found that serious gamers (who played video games 2 hours per day, 3 to 4 days per week, for a minimum of 6 months) held an advantage in sensorimotor learning and hand-eye coordination over nongamers. RT is important for esport competitors as the average professional conducts 500 to 600 actions per minute (LeJacq 2013). In addition, those who participated in video games located visual objects or features on a screen faster, and demonstrated a greater ability in visual mapping, than those who did not play video games (Castel et al. 2005). Experienced gamers had faster RTs to a flashed visual stimulation test, without decreases in accuracy of performance, compared to novice players (Dye et al. 2009). These studies suggest that gaming experience is positively correlated with RT. 
Within traditional athletes, RTs reported in different studies, and under different conditions, varied. In 483 sprinters, those with quicker RTs performed better in a $60 \mathrm{~m}$ sprint than those who had slower RTs (where RT was defined as the time difference between the start signal and the moment the athlete exerted pressure on the starting block) (Gürses and Kamiş 2019). Greater involvement in traditional physical sports activities improved an individual's hand-eye RT and anticipation time responses to LED light cues (Kuan et al. 2018). Interestingly, tennis players displayed faster RTs than both sedentary participants and swimmers when subjected to visual-cue and button-pressing testing (Wang et al. 2013). Even within the same sport, response to different stimuli varied. National Collegiate Athletic Association Division I male soccer players responded faster to a visual command (visible movement $10 \mathrm{~m}$ away) than an auditory one (sound command "Go") (Spierer et al. 2011). Thus, participation in competitive physical sports is associated with improved RTs; especially to visual stimuli.

Little is known about the relative RTs of traditional physical athletes in comparison to esport competitors. This study examined the RTs of college football players (as representatives of traditional physical athletes) and esport competitors. Future studies would then determine whether RTs improve with training, and whether there is a discernible difference in decrease in RTs for competitors training in esports vs. traditional sports. This may lead to training regimens directly associated with improving RT. It was hypothesized that both football players and esport competitors would have faster RTs in visual, auditory, and tactile assessments of RT than the nonactive control group-with esport competitors having the fastest composite RTs (see methods for determination of composite RT).

\section{METHODS AND MATERIALS}

For this IRB-approved study, 3 groups of healthy male college students $(n=12$ per group) were studied: (1) players on the Ohio Northern University football team (NCAA Division III) with at least 2 years of football experience, (2) esport team members at the same college with at least 2 years of gaming experience-ranked between gold and diamond, and (3) a control student group (those who perform little to no physical activity or competitive gaming activity in their daily lives). Although gender has not been observed to influence RT (Woods et al. 2015), this study involved only males. No participant belonged to more than 1 group or had any neurological conditions. Participants were asked to abstain from alcohol, coffee, and energy drinks for 24 hours prior to testing. RTs to visual (color cue test and ruler drop test), auditory (sound cue test), and tactile (probe grabbing test) stimuli were tested in random order. Testing occurred in the esport office at Ohio Northern University, a 4-year regional college located in the Midwest of the United States. For electronic-based tests, a 13-inch 2017 model MacBook Pro $^{\circledR}$ was utilized. Each test was conducted twice using the same device across all participants, and scores were averaged.

\section{RT Tests}

Visual Testing: Visual testing consisted of a computer generated color cue test (RTT 2020) and the ruler drop test. In the color cue test, the participant looked at the computer screen and clicked the computer mouse as fast as he could when the screen switched from red to green. RT was recorded in milliseconds. In the ruler drop test, the participant placed his dominant arm on the table with their hand over the edge of the table in an open " $\mathrm{c}$ " position; the meter stick was held at the midpoint between his thumb and fingertips. As soon as the researcher (sitting opposite to the participant) dropped the ruler, the participant grabbed the meter stick. The vertical distance that the meter stick travelled in centimeters was recorded, then converted into RT $(\mathrm{ms})$ based on the acceleration due to gravity of a free falling object $\left(9.8 \mathrm{~m} / \mathrm{s}^{2}\right)$ provided by the National Aeronautics and SpaceAdministration (NASA 2020): $\mathrm{RT}$ in $\mathrm{ms}=1000 \cdot \sqrt{2 \cdot(\text { distance in } \mathrm{cm} / 100 / 9.8)}$.

Sound Cue: Auditory testing utilized a computer generated test (TYRSS 2020) which was administered by having the participant (with eyes closed) press the spacebar on the testing laptop as soon as he heard a sound from the computer. RT in milliseconds was recorded. Loudness and duration of the signal were consistent across subjects.

Probe Grabbing: Tactile testing consisted of a novel RT test developed by the authors of this study. The researcher hovered a probe $\left(\mathrm{a} \mathrm{Dell}{ }^{\circledR}\right.$ Active Pen-a metallic cylinder approximately $15 \mathrm{~cm}$ long by $0.75 \mathrm{~cm}$ in diameter and weighing $20 \mathrm{~g}$ ) above the participant's open and relaxed dominant hand 
(the participant had his eyes closed); the participant clenched his fist over the probe the moment he felt the probe touch his hand. This process was recorded with a camera at the rate of 60 -frames per second, and RT was measured by analyzing the video frames of the action using Avidemux video editing software (Avidemux 2020). RT was recorded in milliseconds starting from when the probe first touched the participant's hand and ending when the participant's fingers touched his palm.

\section{Statistical Analysis}

All tests were conducted in duplicate and the average of the 2 scores was recorded as the participant score. A composite score (the mean of the 4 tests) was calculated. ANOVA testing was performed for each RT test as well as for the composite scores. Post hoc $t$-tests were performed to determine significant differences between group RTs.

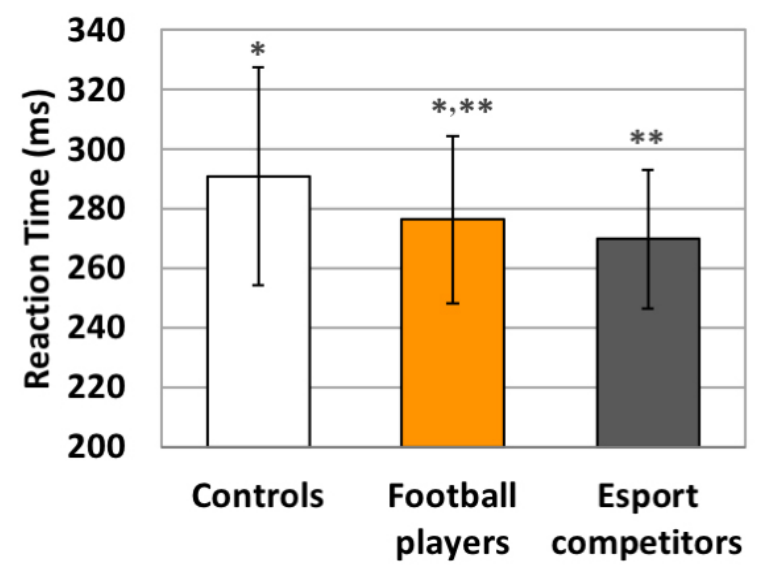

(A)

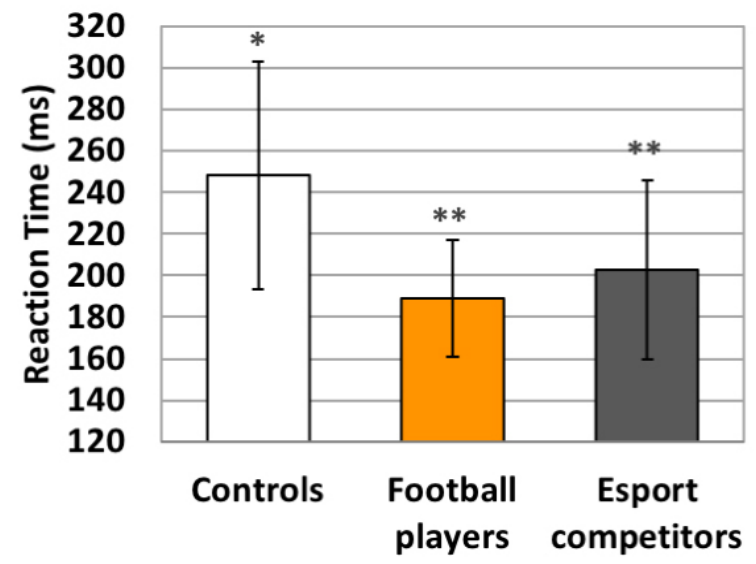

(C)

\section{RESULTS}

All participants were very consistent in their responses. In the color cue test (Fig. 1A), mean RT of esport competitors $(269.83 \pm 23.46 \mathrm{~ms})$ was significantly lower than the control group $(290.83 \pm 36.50 \mathrm{~ms})$, indicating faster RTs. But, RT of esport competitors did not differ significantly from the football players $(276.50 \pm 28.02 \mathrm{~ms})$. In the ruler drop test (Fig. 1B), RT of esport competitors $(174.58 \pm 26.14 \mathrm{~ms})$ and football players $(186.94 \pm 20.30 \mathrm{~ms})$ was significantly faster than the control group $(223.47 \pm 31.11 \mathrm{~ms})$, again, football and esport were not significantly different from each other. In the probe grabbing test (Fig. 1C), esport competitors $(202.84 \pm 42.91$ $\mathrm{ms})$ and football players $(189.08 \pm 28.21 \mathrm{~ms}) \mathrm{had}$ significantly faster RTs than the control group $(248.08 \pm 55 \mathrm{~ms})$. In the sound cue test (Fig. 1D), there was no evidence for a difference between

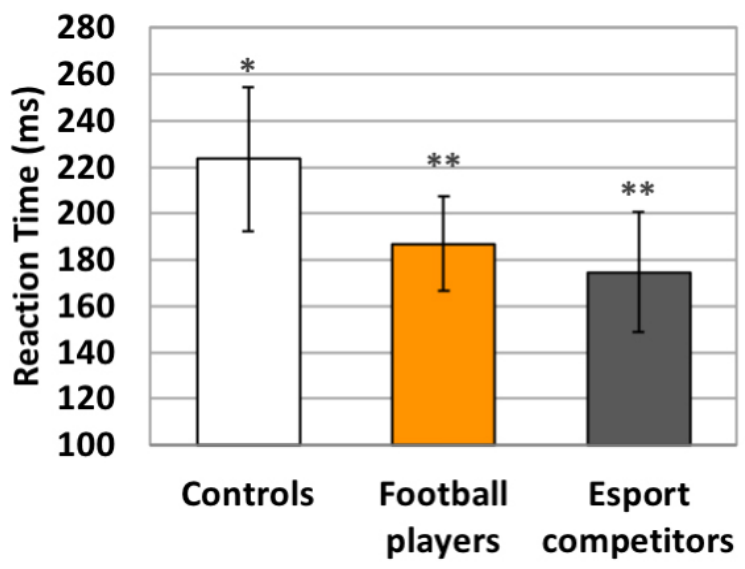

(B)

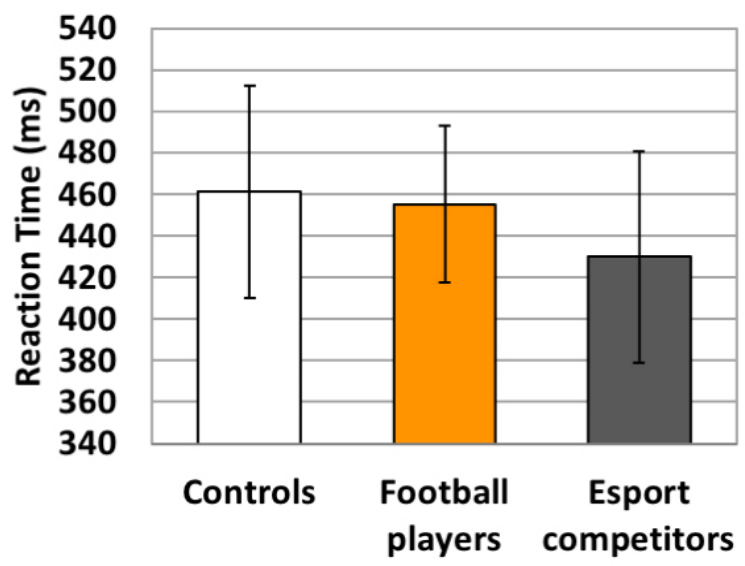

(D)

FIGURE 1. Comparison of mean RTs (ms) of controls (CT), football players (FP), and esport competitors (EC) ( $n=12$ each group). (A) Color cue test. Asterisks indicate significant differences at $p=0.05$ between EC and CT. (B) Ruler drop test. Asterisks indicate significant differences at $p=0.0002$ between EC/FP and CT. (C) Probe grabbing test. Asterisks indicate significant differences at $p=0.0175$ between EC/FP and CT. (D) Sound cue test. No significant differences were observed between EC and FP in any test. 
esport competitors $(429.87 \pm 50.94 \mathrm{~ms})$, football players $(455.33 \pm 37.78 \mathrm{~ms})$, and the control group (461.44 $\pm 51.19 \mathrm{~ms})$.

Overall, esport competitors had the fastest mean composite RT $(269.28 \pm 27.02 \mathrm{~ms})$, followed by football players $(276.96 \pm 17.49 \mathrm{~ms})$ (Fig. 2). The control group $(305.96 \pm 30.5 \mathrm{~ms})$ had the slowest RT. No significant difference was found between esport competitors and football players.

A significant difference was observed in RTs between the 4 tests, with the sound cue test yielding the slowest mean RT (Fig. 3).

RTs of esport competitors and football players were not significantly different, but they both had significantly faster RTs than controls in all but the sound cue test. Involvement in esports or football is associated with faster RTs than nonparticipants, although a causative relationship was not demonstrated in this study.

\section{DISCUSSION}

The significantly faster RTs of esport competitors over the controls in the color cue test supported the findings of Castel et al. (2005), who found that gamers had faster RT to visual stimuli than nongamers. Green et al. (2010) also found faster RTs in esport competitors, using methodology which integrated decision making (not just simple button pressing) in response to stimuli. Their interpretation was that the decreased RTs in gamers playing action games was correlated to feedforward processing. Football players trended toward faster RTs than controls; consistent with the findings of Lesiakowski et al. (2017) that traditional physical athletes had significantly faster RTs in button pressing to a visual cue.

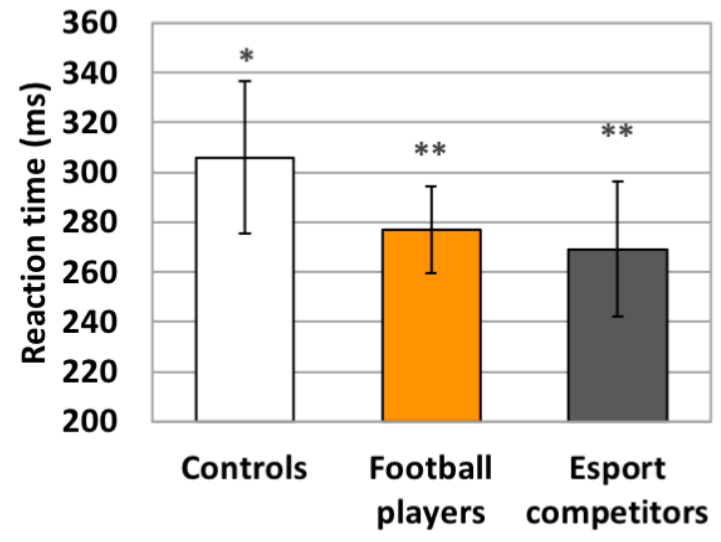

FIGURE 2. Mean composite RTs (ms) of the control group, football players, and esport competitors ( $n=12$ each group). Asterisks indicate significant differences between esport competitors and controls $(p=0.0025)$, and between football players and controls $(p=0.0046)$.
Feedforward processing may also explain why both esport competitors and football players had faster RTs than controls in the ruler drop test. Better hand-eye coordination in response to visual stimuli could possibly be explained by the observation of Mack and Ilg (2014) on their study of gamers: that competitors exhibited faster saccadic peak velocity (eye movement between fixation points) and saccadic RT than noncompetitors. Faster eye movement would give the competitors an advantage responding to a moving visual stimulus, such as a ruler falling. Potentially, video recordings of the ruler drop test and correlated recordings of eye movement might be needed to validate this point.

Probe grabbing was not, however, significantly different between football players and esport competitors. Test analysis could be improved by using a higher quality video recording device that could capture at frame rates higher than 60 per second.

Although the RTs between esport competitors and football players were not significantly different, a trend was seen in that esport competitors had faster RTs than football athletes in all tests except probe grabbing. This trend suggests that different training experiences and activity involvement might influence RT to a specific stimulus.

Whereas Hernández etal. (2005) found no evidence of different RTs to different sensory modalities, RTs in the current study were modality-dependent. Furthermore, in the current study, the auditory based test produced slower RTs than visual based tests, similar to the findings of Spierer et al. (2011) in which soccer players reacted to visual signals faster than to auditory commands. However, these results contradicted a study of nonathletes (medical students

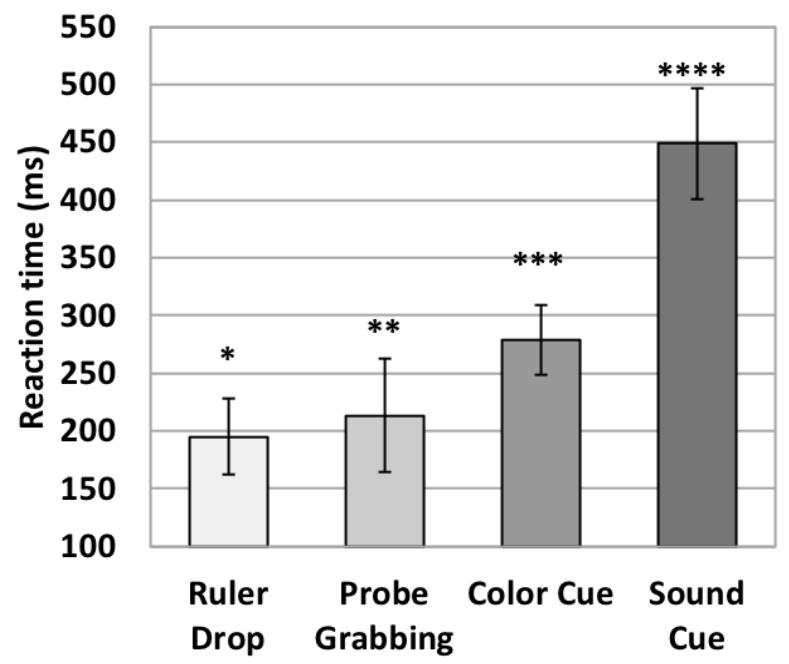

FIGURE 3. RTs (ms) for the 4 tests. Asterisks indicate significant differences by ANOVA. $F_{(140,3)}=286.5, p=0.0000$. 
with various lifestyles) where RTs to auditory cues were actually faster than to visual cues (Jain et al. 2015). In the current study, the color cue test used a red-turning-green signal, similar to the change seen in conventional traffic lights. In contrast, the sound signal for the sound cue test was a unique buzz sound that subjects were unlikely to have heard prior to testing. Lack of difference in RT to sound cue between the 3 groups may have reflected the unfamiliarity of the signal. The faster RT to color cue over sound cue may have been influenced by familiarity to the stimulus, as was observed by Ando et al. (2002). Moreover, during sound cue testing, the subjects in the current study had their eyes closed; this might have delayed their RTs to a degree in comparison to an eyes-open condition (Fuhrman et al. 2015).

It could be inferred that differences in $\mathrm{RT}$ are due to neural processing of incoming stimuli. However, preprocessing signal conduction time might also influence measured RT as auditory stimuli reach the brain in 8 to $10 \mathrm{~ms}$ (Kemp 1973), while visual stimuli take 20 to $40 \mathrm{~ms}$ to reach the occipital cortex (Marshall et al. 1943). In addition, RTs to probe grabbing were significantly faster than those to color cue. Godlove et al. (2014), similarly, found responses to vibration-to-the-hand stimuli were faster than to color-flashing stimuli. Interestingly, the ruler drop test, which incorporated a mobile visual stimulus and hand-eye coordination, had significantly faster RTs than both the color cue test and the probe grabbing test. This was potentially due to the additive effects of seeing the ruler drop and feeling the noncontact pressure generated by its movement. In fact, stimulation with 2 signals, each from a different sensory modality, could result in faster RTs than with a single signal-a phenomenon called coactivation (Miller 1982). Miller's study, however, did not test for tactile stimulation.

The composite RTs assigned each test subject a score which incorporated responses to visual, auditory, and tactile stimuli. This study had a small sample size ( $\mathrm{n}=12$ for each group), which might have contributed to a lack of significant findings in the color cue test (between football players and controls) and the sound cue test. Furthermore, control subjects may have participated in activities with direct correlations to RT which were not considered in this study, such as playing a musical instrument.

\section{CONCLUSION}

Although both esport competitors and football players had faster RTs than the controls, the hypothesis that esport competitors had faster composite RTs than football players was not confirmed-but a supportive trend was evident. This study tested for a positive relationship, but not the causative relationship, between activity involvement and RT. Testing of pre-trained competitors, followed by training and post-training testing, would be needed to ascertain whether activity involvement improved RT or whether fast inherent RT made participants better gamers or athletes. Faster RT may improve safety by facilitating protective maneuvers such as in contact sports, driving, or piloting. As the world becomes more virtually oriented, manipulation of computer peripherals, and the training evinced by repeated use of programs such as competitive video games, may prove beneficial to those whose careers require rapid response (e.g., in firewall protection and cybersecurity, or even technical directors in television studios). Furthermore, those who micromanipulate, such as surgeons performing computer aided techniques, may benefit from the hand-eye coordination associated with competitors in this study.

\section{ACKNOWLEDGEMENTS}

We thank the Department of Biological and Allied Health Sciences of Ohio Northern University for sponsoring this study, Coach Troy Chiefari for providing us access to the esport lounge as the testing room, Coach Dean Paul for helping with recruitment of football athletes, and Jessica Haynie for her contribution to project design.

\section{LITERATURE CITED}

Aktas Y. 2019. Investigation of relationship between reactive agility and cognitive parameters in male football players. J Educ Learn. 8(4):58-63.

https://doi.org/10.5539/jel.v8n4p58

Ando S, Kida N, Oda S. 2002. Practice effects on reaction time for peripheral and central visual fields. Percept Motor Skill. 95(3):747-751.

https://doi.org/10.2466/pms.2002.95.3.747

Avidemux. 2020. [accessed 2020 Feb 6]. Open source video editing and processing software.

Avidemux.sourceforge.net

Bucsuházy K, Semela M. 2017. Case study: reaction time of children according to age. Procedia Engineer. 187:408-413. https://doi.org/10.1016/J.PROENG.2017.04.393 
Castel AD, Pratt J, Drummond E. 2005. The effects of action video game experience on the time course of inhibition of return and the efficiency of visual search. Acta Psychol. $119(2): 217-230$. https://doi.org/10.1016/j.actpsy.2005.02.004

Dean HL, Martí D, Tsui E, Rinzel J, Pesaran B. 2011. Reaction time correlations during eye-hand coordination: behavior and modeling. J Neurosci. 31(7):2399-2412. https://doi.org/10.1523/JNEUROSCI.4591-10.2011

Dye MWG, Green CS, Bavelier D. 2009. Increasing speed of processing with action video games. Curr Dir Psychol Sci. 18(6):321-326. https://doi.org/10.1111/j.1467-8721.2009.01660.x

Fuhrman SI, Redfern MS, Jennings JR, Furman JM. 2015. Interference between postural control and spatial vs. nonspatial auditory reaction time tasks in older adults. J Vestibul Res. 25(2):47-55. https://doi.org/10.3233/ves-150546

Godlove JM, WhaiteEO, Batista AP. 2014. Comparing temporal aspects of visual, tactile, and microstimulation feedback for motor control. J Neural Eng. 11(4):046025. https://doi.org/10.1088/1741-2560/11/4/046025

Gozli DG, Bavelier D, Pratt J. 2014. The effect of action video game playing on sensorimotor learning: evidence from a movement tracking task. Hum Movement Sci. 38:152-162 https://doi.org/10.1016/j.humov.2014.09.004

Green CS, Pouget A, Bavelier D. 2010. Improved probabilistic inference as a general learning mechanism with action video games. Curr Biol. 20(17):1573-1579. https://doi.org/10.1016/j.cub.2010.07.040

Gürses VV, Kamiş O. 2019. The relationship between reaction time and $60 \mathrm{~m}$ performance in elite athletes. J Educ Train Stud. 6(12a):64-69.

https://doi.org/10.11114/jets.v6i12a.3931

Hernández OH, Huchín-Ramirez TC, Vogel-Sprott M. 2005. Behaviorally fractionated reaction time to an omitted stimulus: tests with visual, auditory, and tactile stimuli. Percept Motor Skill. 100(3):1066-1080.

https://doi.org/10.2466/pms.100.3c.1066-1080

Jain A, Bansal R, Kumar A, Singh KD. 2015. A comparative study of visual and auditory reaction times on the basis of gender and physical activity levels of medical first year students. Int J Appl Basic Med Res. 5(2):124-127. https://doi.org/10.4103/2229-516x.157168

Kemp BJ. 1973. Reaction time of young and elderly subjects in relation to perceptual deprivation and signal-on versus signal-off condition. Dev Psychol. 8(2):268-272. https://doi.org/10.1037/h0034147

Kemp S. 1984. Reaction time to a tone in noise as a function of the signal-to-noise ratio and tone level. Percept Psychophys. 36(5):473-476. https://doi.org/10.3758/BF03207501

Kowal M, Toth AJ, Exton C, Campbell MJ. 2018. Different cognitive abilities displayed by action video gamers and non-gamers. Comput Hum Behav. 88:255-262. https://doi.org/10.1016/j.chb.2018.07.010
Kuan YM, Zuhairi NA, Manan FA, Knight VF, Omar R. 2018. Visual reaction time and visual anticipation time between athletes and non-athletes. Malays J Public Health Med. Special volume (1):135-141.

Latorre-Roman PA, Robles-Fuentes A, García-Pinillos F, SalasSánchez J. 2018. Reaction times of preschool children on the ruler drop test: a cross-sectional study with reference values. Percept Motor Skill. 125(5):866-878. https://doi.org/10.1177/0031512518789563

LeJacq Y. 2013 Oct 24. How fast is fast? Some pro gamers make 10 moves per second. NBC News Digital. [accessed 2020 Aug 4]. https:/www.nbcnews.com/technolog/how-fastfast-some-pro-gamers-make-10-moves-second-8c11422946

Lesiakowski P, Krzepota J, Zwierko T. 2017. The differentiation of visual sensorimotor processes in the representatives of various sport disciplines. Cent Eur J Sport Sci Med. 19(3):43-53.

https://doi.org/10.18276/cej.2017.3-04

Mack DJ, Ilg UJ. 2014. The effects of video game play on the characteristics of saccadic eye movements. Vision Res. 102:26-32. https://doi.org/10.1016/j.visres.2014.07.010

Marshall WH, Talbot SA, Ades HW. 1943. Cortical response of the anaesthesized cat to gross photic and electrical afferent stimulation. J Neurophysiol. 6(1):1-15. https://doi.org/10.1152/jn.1943.6.1.1

Miller J. 1982. Divided attention: evidence for coactivation with redundant signals. Cognitive Psychol. 14(2):247-279. https://doi.org/10.1016/0010-0285(82)90010-x

[NASA] National Aeronautics and Space Administration. 2020. Motion of free falling object. [accessed 2020 Feb 6] https:/www.grc.nasa.gov/www/k-12/airplane/mofall.html

Radák Z. 2018. The physiology of physical training. London (UK): Academic Press (an imprint of Elsevier). 280 p. https://doi.org/10.1016/C2017-0-01911-0

[RTT] Reaction time test. 2020. [accessed 2020 Feb 6]. Online interactive test. https:/www.humanbenchmark.com/tests/reactiontime

Spierer DK, Petersen RA, Duffy K. 2011. Response time to stimuli in Division I soccer players. J Strength Cond Res. 25(4):1134-1141. https://doi.org/10.1519/jsc.0b013e3181d09e4c

[TYRSS] Test your reaction speed to sound. 2020. [accessed 2020 Feb 6]. Online interactive test. https://playback.fm/audio-reaction-time

Wang CH, Chang CC, Liang YM, Shih CM, Muggleton NG, Juan CH. 2013. Temporal preparation in athletes: a comparison of tennis players and swimmers with sedentary controls. J Motor Behav. 45(1):55-63. https://doi.org/10.1080/00222895.2012.740522

Woods DL, Wyma JM, Yund EW, Herron TJ, Reed B. 2015. Factors influencing the latency of simple reaction time. Front Hum Neurosci. 9(article 131):1-12. https://doi.org/10.3389/fnhum.2015.00131 